\title{
Can Cancer Survivors Donate Convalescent Plasma for the Treatment of COVID-19?
}

\author{
Ajit Venniyoor $^{1}$ \\ ${ }^{1}$ National Oncology Centre, The Royal Hospital, Muscat, Sultanate \\ of Oman
}

Ind J Med Paediatr Oncol 2021;42:21-24.

\section{Case Vignette}

SS is a 50-year-old female treated for stage III colon cancer in 2010 and on a 6-monthly follow-up. She missed her scheduled review in July 2020 and informed that she could not come on for her appointment due to coronavirus disease 2019 (COVID-19) infection, which affected her whole family, all of whom had mild symptoms and recovered uneventfully. I suggested that she could now donate convalescent plasma (CP) for the treatment of seriously ill COVID-19 patients, and she was immediately interested. I advised her to go to the hospital's blood transfusion center and volunteer. Later, I had a call from the transfusion medical officer asking me to clarify whether cancer survivors can donate blood or blood products.

\section{Introduction}

Severe acute respiratory syndrome-coronavirus-2 (SARS-CoV-2), the cause of COVID-19, is the latest virus in the long line of infectious agents that have challenged human beings through our history. ${ }^{1}$ The pandemic started with an outbreak of pneumonia of unknown origin in Wuhan in the Hubei province of China in December 2019. By January 7, 2020 , Chinese scientists had isolated the causative novel coronavirus (CoV). The Chinese flu spread rapidly through China and was exported, initially to Thailand, Japan, Korea, the United States, Vietnam, and Singapore, and later to the rest of the world. ${ }^{2}$ As of August 03, 2020, there are over 18 million recorded cases with $\sim 700,000$ deaths in 213 countries (www.worldometer.info).

The methods of managing the COVID-19 patients have varied widely across the world and continue to evolve.

There is a tacit understanding that the final control over the virus will be achieved only when there is herd immunity, either by active infection or by a vaccine (the exact percentage of immune population required for this remains a matter

\author{
Address for correspondence Ajit Venniyoor, MRCP, DM, National \\ Oncology Centre, The Royal Hospital, Post Box 1331, Seeb, \\ Postal Code 111, Muscat, Sultanate of Oman \\ (e-mail: avenniyoor@gmail.com).
}

of controversy). In the meantime, prevention by measures such as social distancing, hand washing, and face masks has been recommended ${ }^{3}$ to "flatten the curve," thus spreading out the cases, and avoid overwhelming the health-care facilities by unmanageable spikes in symptomatic cases needing active treatment. The role of pharmacological agents in prevention, such as hydroxychloroquine, is controversial.

Treatment of symptomatic cases depends on the severity, and in addition to the management of complications, is a two-pronged approach-antiviral agents (only remdesivir has a high level of evidence ${ }^{4}$ ) and anti-inflammatory drugs (again, only dexamethasone has a high level of evidence ${ }^{5}$ ). An indirect way of targeting the virus is by boosting host immunity with the use of $\mathrm{CP}$.

\section{Convalescent Plasma}

Passive antibody therapy through transfusion of $\mathrm{CP}$ is a short-term strategy for conferring immediate immunity and has been used in the past for similar viral epidemics such as Middle East respiratory syndrome and SARS- 1 . Readers are referred to an excellent review by Bloch et $\mathrm{al}^{6}$ for details of this therapy. CP (or immune) is a plasma containing neutralizing antibodies (NAbs) to a specific infection collected from previously infected individuals after resolution of infection. Many of the initial studies displaying the positive effects of $\mathrm{CP}$ have been from China. A systematic review ${ }^{7}$ of five studies (four from China) confirmed the beneficial effects in almost all patients, including a rise in NAb titers and disappearance of circulating CoV-2 RNA, and possible reduction in mortality. However, a large Cochrane analysis ${ }^{8}$ of $\mathrm{CP}$ reported that the safety and efficacy of CP in COVID-19 patients were "uncertain," and that data from the ongoing 98 studies (including 50 randomized trials) are awaited.

The CP donor must have had a molecularly confirmed COVID-19 infection (such as real-time polymerase chain
DOI https://doi.org/ $10.1055 / \mathrm{s}-0041-1729734$ ISSN 0971-5851
(C) 2021. Indian Society of Medical and Paediatric Oncology This is an open access article published by Thieme under the terms of the Creative Commons Attribution-NonDerivative-NonCommercial-License, permitting copying and reproduction so long as the original work is given appropriate credit. Contents may not be used for commercial purposes, or adapted, remixed, transformed or built upon. (https://creativecommons.org/licenses/by-nc-nd/4.0/). Thieme Medical and Scientific Publishers Pvt. Ltd. A-12, 2nd Floor, Sector 2, Noida-201301 UP, India 
reaction positivity of nasopharyngeal swab), recovery from symptoms of at least 14 days, and a negative test subsequently (to prevent transmission to blood transfusion department staff).

However, the last criterion is not absolute as it is accepted that most cases of mild infection will clear the virus by the 10 th day.

A Chinese study ${ }^{9}$ of 175 patients who recovered from mild COVID-19 infection showed that most COVID-19 patients developed SARS-CoV-2-specific NAbs by the convalescent phase of infection. However, 30\% had low titers and 10 of them had undetectable levels-it was not clear how they recovered without generating an antibody response.

The titers of NAbs reached their peak at 10 to 15 days after the disease onset and that antibodies targeting on different domains of S protein, including S1, receptor-binding domain (RBD), and S2, contributed to virus neutralization. These and other studies ${ }^{10}$ indicate that seroconversion of the donor occurs between day 8 and 21 after the onset of symptoms, and peak antibody titers are obtained 14 days after the resolution of symptoms. The relationship between total and NAbs is not clear.

Theoretically, the CP should be tested for antibody titers before transfusion, but tests for specific NAbs are not generally available and quantitative assays for total antibodies (such as ELISA) have not yet been validated. The Indian government guidelines ${ }^{11}$ suggest that in the case of nonavailability of tests for NAbs, plasma immunoglobulin G titer (against S-protein RBD) above 1:640 should be used.

The collection and administration of $\mathrm{CP}$ follow standard procedure, but it is recommended that apheresis be used rather than whole blood donation; this will yield 400 to $800 \mathrm{~mL}$ of plasma, enough for two to four units of CP. ${ }^{6}$ The units are frozen after collection while awaiting standard donor testing such as for other infections. A typical patient, usually critically ill, needs only one or two units of CP for treatment. This should provide adequate titers that last from few weeks to few months. ${ }^{6}$

The transient nature of antibody response is a matter of concern. A recent letter to the New England Journal of Medicine ${ }^{12}$ suggests that there is a rapid fall in antibody titers (half-life of 36 days) after recovery from mild infection-this may not be important for the survivor as reexposure could result in rapid recovery due to memory B cells, but suggests that $\mathrm{CP}$ may not contain adequate antibody titers if collection is delayed. Another paper from Kings College, London, ${ }^{13}$ reported that the antibodies appear in $>95 \%$ of cases after 8 days of symptoms, but levels began to fall about a month later, sometimes to nearly undetectable levels. Hence, the window of opportunity for donation and the donor pool could be smaller than estimated.

\section{Cancer and COVID-19}

The risk factors associated with the severity of infection and mortality of COVID-19 infection include male sex, age, obesity, comorbid medical conditions (diabetes, chronic obstructive pulmonary disease, and chronic kidney failure), and cancer. The initial ${ }^{14}$ and subsequent papers ${ }^{15,16}$ from China indicated an increased severity and mortality in cancer patients, leading to extraordinary changes in their management. ${ }^{17}$ However, more recent papers ${ }^{18,19}$ suggest that the risk is exaggerated and may be limited to specific subsets, such as those with hematological malignancies and those on immunotherapy. ${ }^{20}$ Indeed, there is a possibility that prostate cancer patients on androgen deprivation therapy may actually be protected, ${ }^{21}$ consistent with epidemiological data that women are less affected (it would be interesting to see whether patients at the other end of the spectrum, i.e., postmenopausal women with breast cancer on aromatase inhibitor therapy are more at risk).

\section{Blood Donation by Cancer Patients}

Blood donation by cancer survivors is a matter of controversy due to a theoretical risk of transmitting cancer by circulating tumor cells. Donor-transmitted cancer has been reported in recipients of organ transplantation ${ }^{22}$; however, cancer transmission through donation of blood or blood products is unknown..$^{23}$ Large vein-to-vein databases are maintained to monitor the health of donors and specific recipients for infectious and noninfectious early and late complications, ${ }^{24}$ which have not reported any such transmission. For instance, the Scandinavian Donations and Transfusions database examined 354,094 transfused patients, of whom 3\% received at least one blood unit from a precancerous donor (defined as someone who developed a cancer within 5 years of donating blood). There was no difference in the cancer incidence or site of cancer in them, compared with those who received blood donation from those who remained cancer free in those 5 years. ${ }^{25}$

In spite of such consistent data, the guidelines allowing cancer patients to donate blood vary by country and organization. The Joint United Kingdom Blood Transfusion and Tissue Transplantation Services permits only those with treated basal cell carcinoma or in situ cancer or premalignant conditions (such as polyposis coli) to donate blood. ${ }^{26}$ On the other hand, most hospitals in the United States permit blood donation if the survivor has been off treatment for $>12$ months without recurrence. However, hematological malignancies (leukemias, lymphomas, and myeloma) bring with it a lifelong bar on donation. This is in line with the recommendations of the American Red Cross. ${ }^{27}$ Hence, there is no reason why cancer and COVID-19 survivors (double survivors) should not donate $\mathrm{CP}$. However, the Indian guideline $^{28}$ in their donor selection criteria state that malignancy is a reason for permanent deferment-there is an urgent need to reexamine this.

\section{Antibody Titers in Cancer Survivors}

As of January 2019, it is estimated that there are 16.9 million cancer survivors in the United States, representing $5.0 \%$ of the population. ${ }^{29}$ This is a heterogeneous population and 
at higher risk for second primary malignancy, both due to inherited genetic defects and effects of cancer drugs, and with long-term immune abnormalities. Immunization schedule for cancer patients and survivors is different from that of general population and includes contraindication to live vaccines. ${ }^{30,31}$ It is assumed that cancer and its treatment could lead to lowered immunity, which raises the question whether COVID-19/ cancer survivors can generate sufficient antibody titers. At present there is no direct data on this, but as recent papers indicate equivalent survival in cancer patients affected by $\mathrm{CoV},{ }^{19}$ it can be assumed that they too generate an adequate antibody response.

Poor antibody responses to vaccines are common in patients of hematological malignancies, especially those who receive anti-B cell agents such as rituximab. ${ }^{32}$ Among patients with Hodgkin lymphoma, for example, vaccine responses remain impaired for as long as 7 years after treatment. ${ }^{33}$ However, such survivors of hematological malignancies are automatically excluded from CP donation. On the other hand, patients with solid tumors are classically given myelosuppressive rather than immunosuppressive therapy. Hence, the degree of immunosuppression is expected to be minimal. Consistent with this, no deficiency in serologic response was noted in patients with solid tumors, such as gastric and colorectal cancers, vaccinated with pneumococcal vaccine, ${ }^{34}$ or influenza vaccine..$^{35}$

They have responded with adequate antibody titers. Thus, both direct and indirect evidence suggests that survivors of solid tumors who recovered from COVID-19 will have equivalent NAbs. However, this requires confirmation.

\section{Conclusions}

Cancer survivors who survive COVID-19 (double survivors) are expected to have sufficient NAb titers in their plasma to be considered for CP donation. The risk of transmitting cancer through blood or blood products is negligible to nonexistent. Recent data indicate that patients recovering from mild CoV infection (representing $85 \%$ of the infected population) have a limited period for donating their plasma due to rapid decay of antibody titers; this suggests that the donor pool is smaller than estimated. Cancer survivors form a not-so-negligible percentage of the population and they are well aware of the importance of blood transfusion by personal experience.

Consequently, they are well motivated to donate. It can be concluded that CP donation by survivors of solid tumors who are free of recurrences for at least 12 months after treatment is safe and of equivalent efficacy. In the meantime, there is an urgent need to revise the guidelines by the Indian regulatory authorities banning donation of blood and blood products by cancer survivors.

\section{Financial Support and Sponsorship \\ Nil.}

\section{Conflicts of Interest}

There are no conflicts of interest.

\section{References}

1 Morens DM, Daszak P, Markel H, Taubenberger JK. Pandemic COVID-19 joins history's pandemic legion. MBio 2020;11(3):e00812-e00820

2 Kulkarni P, Dhanushkodi M. Novel coronavirus severe acute respiratory syndrome-coronavirus 2 (coronavirus disease 2019): we shall overcome! Indian J Med Paediatr Oncol 2020;41:110-113

3 MacIntyre CR, Wang Q. Physical distancing, face masks, and eye protection for prevention of COVID-19. Lancet 2020;395(10242):1950-1951

4 Horby P, Lim WS, Emberson JR, et al. .RECOVERY Collaborative Group. Dexamethasone in hospitalized patients with Covid-19-preliminary report. N Engl J Med 2020;:NEJMoa202143610.1056/ NEJMoa2021436

5 Beigel JH, Tomashek KM, Dodd LE, et al. Remdesivir for the treatment of Covid-19-preliminary report. N Engl J Med 2020;383:992-994 [doi: 10.1056/NEJMoa2007764]

6 Bloch EM, Shoham S, Casadevall A, et al. Deployment of convalescent plasma for the prevention and treatment of COVID-19. J Clin Invest 2020;130(6):2757-2765

7 Rajendran K, Krishnasamy N, Rangarajan J, Rathinam J, Natarajan M, Ramachandran A. Convalescent plasma transfusion for the treatment of COVID-19: systematic review. J Med Virol 2020;92(9):1475-1483 [doi: 10.1002/jmv.25961]

8 Piechotta V, Chai KL, Valk SJ, et al. Convalescent plasma or hyperimmune immunoglobulin for people with COVID19: a living systematic review. Cochrane Database Syst Rev 2020;7:CD013600

9 prpt. Wu F, Wang A, Liu M, et al. Neutralizing antibody responses to SARS-CoV-2 in a COVID-19 recovered patient cohort and their implications. medRxiv 2020/prpt

10 Long QX, Liu BZ, Deng HJ, et al. Antibody responses to SARSCoV-2 in patients with COVID-19. Nat Med 2020;26(6):845-848

11 Anonymous. Clinical Management Protocol: COVID19. Ministry of Health and Family Welfare Guidelines. Available from: https://www.mohfw.gov.in/pdf/ Clinical ManagementProtocolforCOVID19.pdf. Accessed April 13, 2021

12 Ibarrondo FJ, Fulcher JA, Goodman-Meza D, et al. Rapid decay of anti-SARS-CoV-2 antibodies in persons with mild COVID19. N Engl J Med 2020;383(11):1085-1087

13 prpt. Seow J, Graham C, Merrick B, et al. Longitudinal evaluation and decline of antibody responses in SARSCoV-2 infection. medRxiv 2020. pre-print. [doi: 10.1101/2020.07.09.20148429]./prpt

14 Yu J, Ouyang W, Chua MLK, Xie C. SARS-CoV-2 transmission in patients with cancer at a tertiary care hospital in Wuhan, China. JAMA Oncol 2020;6(7):1108-1110

15 Dai M, Liu D, Liu M, et al. Patients with cancer appear more vulnerable to SARS-CoV-2: a multi center study during the COVID-19 outbreak. Cancer Discov 2020;10(6):783-791

16 Liang W, Guan W, Chen R, et al. Cancer patients in SARSCoV-2 infection: a nationwide analysis in China. Lancet Oncol 2020;21(3):335-337

17 Pathak R, Chitkara G, Kulkarni P, Bhattacharyya GS, Gupta S, Bajpai J. Tale of constant adaptation, revision, and change of cancer therapy during the ongoing COVID crisis: adapting to cope and win. Indian J Med Paediatr Oncol 2020;41:103-109

18 van Dam PA, Huizing M, Mestach G, et al. SARS-CoV-2 and cancer: are they really partners in crime? Cancer Treat Rev 2020;89:102068

19 Lee LY, Cazier JB, Angelis V, et al. UK Coronavirus Monitoring Project Team. COVID-19 mortality in patients with cancer on chemotherapy or other anticancer treatments: a prospective cohort study. Lancet 2020;395(10241):1919-1926 
20 prpt . Robilotti EV, Babady NE, Mead PA, et al. Determinants of severity in cancer patients with COVID-19 illness. medRxiv 2020. pre-print [doi: 10.1101/2020.05.04.20086322]./prpt

21 Montopoli M, Zumerle S, Vettor R, et al. Androgen-deprivation therapies for prostate cancer and risk of infection by SARSCoV-2: a population-based study $(\mathrm{N}=4532)$ Ann Oncol 2020;31(8):1040-1045

22 Desai R, Collett D, Watson CJ, Johnson P, Evans T, Neuberger J. Cancertransmissionfromorgandonors-unavoidablebutlowrisk. Transplantation 2012;94(12):1200-1207

23 Yang H, Lee J, Seed CR, Keller AJ. Can blood transfusion transmit cancer? A literature review. Transfus Med Rev 2010;24(3):235-243

24 Goel R, Tobian AA, Shaz BH. Noninfectious transfusion-associated adverse events and their mitigation strategies. Blood 2019;133(17):1831-1839

25 Edgren G, Hjalgrim H, Reilly M, et al. Risk of cancer after blood transfusion from donors with subclinical cancer: a retrospective cohort study. Lancet 2007;369(9574):1724-1730

26 Anonymous. Joint United Kingdom (UK) Blood Transfusion and Tissue Transplantation Services Professional Advisory Committee Donor Selection Guidelines. Available from: https:// www.transfusionguidelines.org/dsg/wb/guidelines/ ma006-malignancy. Accessed April 13, 2021

27 Anonymous. American Red Cross. Frequently Asked Questions. Available from: https://www.redcrossblood.org/faq. html\#eligibility-medicalconditions. Accessed April 13, 2021

28 Anonymous. National Blood Transfusion Council. Guidelines for Blood Donor Selection and Donor Referrals. Available from: http://nbtc.naco.gov.in/page/policies_guidelines/. Accessed April 13, 2021

29 Miller KD, Nogueira L, Mariotto AB, et al. Cancer treatment and survivorship statistics, 2019. CA Cancer J Clin 2019;69(5):363-385

30 Rubin LG, Levin MJ, Ljungman P, et al. Infectious Diseases Society of America. 2013 IDSA clinical practice guideline for vaccination of the immunocompromised host. Clin Infect Dis 2014;58(3):e44-e100

31 Shah MK, Kamboj M. Immunizing cancer patients: which patients? Which vaccines? When to give? Oncology (Williston Park) 2018;32(5):254-258, C3

32 Nazi I, Kelton JG, Larché M, et al. The effect of rituximab on vaccine responses in patients with immune thrombocytopenia. Blood 2013;122(11):1946-1953

33 Frederiksen B, Specht L, Henrichsen J, Pedersen FK, Pedersen-Bjergaard J. Antibody response to pneumococcal vaccine in patients with early stage Hodgkin's disease. Eur J Haematol 1989;43(1):45-49

34 Choi W, Kim JG, Beom SH, et al. Immunogenicity and optimal timing of 13-valent pneumococcal conjugate vaccination during adjuvant chemotherapy in gastric and colorectal cancer: a randomized controlled trial. Cancer Res Treat 2020;52(1):246-253

35 Nordøy T, Aaberge IS, Husebekk A, et al. Cancer patients undergoing chemotherapy show adequate serological response to vaccinations against influenza virus and Streptococcus pneumoniae. Med Oncol 2002;19(2):71-78 